Augusto Cesar Arenaro e Mello Dias

\title{
Posicionamento Competitivo Dentro dos Grupos Estratégicos da Indústria Farmacêutica Brasileira
}

Dissertação apresentada como requisito parcial para obtenção do grau de Mestre pelo Programa de Pós-Graduação em Administração de Empresas da PUC-Rio.

Orientador: Prof. Jorge Ferreira da Silva

Rio de Janeiro

Julho de 2005 


\section{Augusto Cesar Arenaro e Mello Dias}

\section{Posicionamento Competitivo Dentro dos Grupos Estratégicos da Indústria Farmacêutica Brasileira}

Dissertação apresentada como requisito parcial para obtenção do grau de Mestre pelo Programa de Pós-Graduação em Administração de Empresas da PUC-Rio. Aprovada pela Comissão Examinadora abaixo assinada.

Prof. Jorge Ferreira da Silva Orientador Departamento de Administração - PUC-Rio

Prof. Paulo Cesar de Mendonça Motta Departamento de Administração - PUC-Rio

Prof. Moisés Balassiano EBAPE - FGV

Prof. João Pontes Nogueira Vice-Decano de Pós-Graduação do CCS 
Todos os direitos reservados. É proibida a reprodução total ou parcial do trabalho sem a autorização da universidade, do autor e do orientador.

\section{Augusto Cesar Arenaro e Mello Dias}

Graduou-se em Engenharia Elétrica pela Universidade do Estado do Rio de Janeiro (UERJ) em 1985. Cursou o MBA Management no IAG da PUC-Rio em 2003. Atualmente é gerente geral da Ranova Atacadista, distribuidora atuante no varejo de ferragens e materiais de construção, onde trabalha há dezesseis anos.

Ficha Catalográfica

Dias, Augusto Cesar Arenaro e Mello

Posicionamento competitivo dentro dos grupos estratégicos da indústria farmacêutica brasileira / Augusto Cesar Arenaro e Mello Dias ; orientador: Jorge Ferreira da Silva. - Rio de Janeiro : PUC, Departamento de Administração, 2005.

164 f. ; $30 \mathrm{~cm}$

Dissertação (mestrado) - Pontifícia Universidade Católica do Rio de Janeiro, Departamento de Administração.

Inclui referências bibliográficas.

1. Administração - Teses. 2. Estratégia. 3. Grupos estratégicos. 4. Tipologia de Miller e Dess. 5. Posicionamento competitivo. 6 . Indústria farmacêutica. I. Silva, Jorge Ferreira da. II. Pontifícia Universidade Católica do Rio de Janeiro. Departamento de Administração. III. Título. 


\section{Agradecimentos}

Ao Professor Jorge Ferreira da Silva por sua orientação e contribuição para o aperfeiçoamento de minha formação acadêmica ao longo de todo o curso e, especialmente, na realização deste trabalho.

Aos meus amigos Alexandre Zílio, Gilberto Mesquita e Valéria Chaves, grandes parceiros do MBA, pelo companheirismo, apoio e colaboração nos diversos trabalhos que juntos realizamos.

Aos meus pais, Manoel Cesar e Sonia Maria, pelo amor e carinho dedicados ao longo de toda a minha vida.

A minhas filhas, Ana Carolina e Juliana, pelo entendimento da necessidade da ausência do pai em diversos momentos de lazer devido às variadas tarefas exigidas pelo curso.

A minha esposa, companheira e amiga Claudia Marcia por todo o amor, apoio e compreensão durante os nossos dezesseis anos de casamento, mas, especialmente, na dura jornada dos últimos anos.

E, por último, o meu agradecimento especial a minha avó materna (in memorian), Maria da Luz, por seu amor, dedicação e exemplo de vida que contribuíram, de forma inestimável, para a minha formação como ser humano. 


\section{Resumo}

Dias, Augusto Cesar Arenaro e Mello; Silva, Jorge Ferreira da (Orientador). Posicionamento competitivo dentro dos grupos estratégicos da indústria farmacêutica brasileira. Rio de Janeiro, 2005. 164p. Dissertação de Mestrado - Departamento de Administração, Pontifícia Universidade Católica do Rio de Janeiro.

Diferentemente de várias pesquisas que estudam as diferenças de desempenho somente entre grupos estratégicos para verificar as implicações nos resultados das empresas devido ao posicionamento estratégico adotado, este estudo tem por objetivo examinar tais diferenças não só entre, mas também dentro dos grupos estratégicos para responder à esta mesma questão. Utilizando um banco de dados com informações da indústria farmacêutica brasileira no período de 1999 a 2002, esta pesquisa toma por base o estudo de Miller e Dess sobre tipologias para identificar os grupamentos existentes e as estratégias relevantes neste segmento industrial, buscando relacionar o posicionamento competitivo utilizado pelas empresas ao desempenho observado. Os resultados obtidos sugerem que os melhores desempenhos pertencem aos grandes laboratórios estrangeiros que fabricam medicamentos de marca e que utilizam estratégias simultâneas de baixo custo e diferenciação em um escopo amplo de atuação de mercado. Estes resultados mostram ainda indícios de que a tipologia de Miller e Dess descreve o posicionamento estratégico das empresas dentro da indústria farmacêutica brasileira melhor do que a tipologia de Porter. Por fim, o estudo sugere que nenhuma empresa possui vantagem competitiva pelo fato de estar posicionada como principal, secundária ou solitária dentro dos grupos estratégicos desta indústria.

\section{Palavras-chave}

Estratégia; grupos estratégicos; tipologia de Miller e Dess; posicionamento competitivo; indústria farmacêutica. 
Abstract

Dias, Augusto Cesar Arenaro e Mello; Silva, Jorge Ferreira da (Advisor). Competitive positioning within the strategic groups of the Brazilian pharmaceutical industry. Rio de Janeiro, 2005. 164p. MSc. Dissertation - Departamento de Administração, Pontifícia Universidade Católica do Rio de Janeiro.

Unlike many researches that study performance differences only among strategic groups to verify the implications on firms results due to their strategic positioning, this study aims to examine such differences not only among, but also within strategic groups in order to answer the same question. Using a database that contains information about the Brazilian pharmaceutical industry from 1999 to 2002, this research employs Miller and Dess' study about typologies as a base to identify existing groups and their relevant strategies in this industrial sector, and to relate the competitive positioning of firms to their achieved performance. Obtained results suggest that the best performances belong to foreign giant laboratories that produce branded drugs and that simultaneously utilize low cost and differentiation strategies with a broad market orientation. Furthermore, the aforementioned attained results show strong indicia that Miller and Dess' typology describes strategic positioning of firms within the Brazilian pharmaceutical industry better than Porter's typology does. Finally, this study suggests that no company has competitive advantage because of its positioning as a core, secondary or solitary firm within the strategic groups of this industry.

\section{Keywords}

Strategy; strategic groups; Miller and Dess' typology; competitive positioning; pharmaceutical industry. 


\section{Sumário}

1. Definição do Problema 14

1.1. Introdução 14

1.2. Objetivos 17

1.2.1. Objetivo Final 17

1.2.2. Objetivos Intermediários $\quad 17$

1.3. Delimitação do Estudo 17

1.4. Relevância do Estudo 18

2. Referencial Teórico 19

2.1. Ambiente Competitivo 19

2.1.1. Modelo das Cinco Forças de Porter 20

2.2. Conceito de Estratégia 25

2.3. Estratégias Competitivas 26

2.4. Grupos Estratégicos 27

2.5. Heterogeneidade da Indústria e de Grupos

Estratégicos $\quad 29$

2.6. Diferenças de Desempenho Entre Grupos

Estratégicos 30

2.7. Diferenças de Desempenho Dentro de Grupos

Estratégicos 31

2.8. Critérios de Avaliação do Desempenho 33

2.9. Tipologias Estratégicas 34

2.9.1. Tipologia de Porter 35

2.9.2. Tipologia de Miller e Dess 39

3. Visão Geral do Mercado Farmacêutico 44

3.1. Introdução 44

3.2. Definições 45

3.3. Aspectos Técnicos 47

3.4. Mercado Mundial 48 
3.5. Mercado Brasileiro

3.6. Medicamentos Genéricos 53

3.6.1. Introdução 53

3.6.2. Mercado Mundial 54

3.6.3. Mercado Brasileiro 54

4. Metodologia 57

4.1. Tipo de Pesquisa 57

4.2. Universo e Amostra 57

4.3. Coleta de Dados 59

4.3.1. Seleção das Variáveis Estratégicas 59

4.3.2. Seleção das Variáveis de Desempenho 62

4.4. Tratamento dos Dados 64

4.5. Limitações do Método 68

5. Apresentação e Análise dos Resultados 69

5.1. Análise da Normalidade das Variáveis 69

5.2. Análise de Correlações Entre as Variáveis 70

5.3. Redução do Espaço Estratégico 74

5.4. Formação dos Grupos Estratégicos 78

5.5. Análise dos Grupos Estratégicos 84

5.5.1. Grupo I: Diferenciação + Baixo Custo + Escopo Amplo 85

5.5.2. Grupo II: Diferenciação + Baixo Custo + Escopo Estreito $\quad 87$

5.5.3. Grupo III: Diferenciação + Escopo Amplo 88

5.5.4. Grupo IV: Diferenciação + Escopo Estreito 88

5.5.5. Grupo V: Baixo Custo + Escopo Amplo 89

5.5.6. Grupo VI: Baixo Custo + Escopo Estreito 90

5.5.7. Grupo VII: Sem Posicionamento 91

5.5.8. Comparação com os Grupos Estratégicos

Formados à Luz da Tipologia de Porter 92

5.6. Comparação de Desempenho Entre Grupos 
5.7. Posicionamento Competitivo Dentro dos Grupos

Estratégicos

5.7.1. Grupo I: Diferenciação + Baixo Custo +

Escopo Amplo

102

5.7.2. Grupo II: Diferenciação + Baixo Custo +

Escopo Estreito

103

5.7.3. Grupo III: Diferenciação + Escopo Amplo 104

5.7.4. Grupo IV: Diferenciação + Escopo Estreito 105

5.7.5. Grupo V: Baixo Custo + Escopo Amplo 106

5.7.6. Grupo VI: Baixo Custo + Escopo Estreito 107

5.7.7. Grupo VII: Sem Posicionamento 108

5.8. Comparação de Desempenho Segundo o

Posicionamento 110

5.8.1. Dentro dos Grupos Estratégicos 110

5.8.2. Dentro da Indústria Farmacêutica 112

5.9. Construção de Modelo para Previsão de

Desempenho 114

5.9.1. Determinação dos Componentes do Modelo 1

5.9.2. Análise Residual do Modelo 1

5.9.3. Determinação dos Componentes do Modelo 2

5.9.4. Análise Residual do Modelo 2

6. Conclusões e Sugestões 120

6.1. Conclusões Sobre os Resultados da Pesquisa 120

6.2. Sugestões para Futuras Pesquisas 124

7. Referências Bibliográficas 126

8. Anexos 130

8.1. Estatísticas Descritivas da Base de Dados Original $\quad 130$

8.2. Análise de Normalidade das Variáveis 130

8.2.1. Testes de Kolmogorov-Smirnov e Shapiro-Wilk 130

8.2.2. Histogramas e Gráficos das Variáveis

Estratégicas 
8.2.3. Histogramas e Gráficos das Variáveis de

Desempenho

8.3. Análise de Correlações 136

8.3.1. Variáveis Estratégicas 136

8.3.2. Variáveis de Desempenho 137

8.4. Análise de Fatores 138

8.5. Análise Multivariada dos Fatores 140

8.6. Análise de Cluster K-Means 141

8.7. Comparação dos Centróides - Wilcoxon Signed

Ranks Test 143

8.8. Análise Multivariada do Desempenho Entre Grupos 144

8.9. Análise de Normalidade da Distância ao Centro do

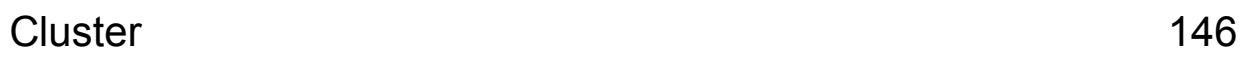

8.10. Análise Multivariada do Desempenho Dentro dos

Grupos 147

8.10.1. Grupo I 147

8.10.2. Grupo II 149

8.10.3. Grupo V 151

8.10.4. Grupo VI 153

8.10.5. Grupo VII 155

8.11. Análise Multivariada do Desempenho Dentro da Indústria 157

8.12. Análise de Regressão do Modelo 1

8.13. Análise de Regressão do Modelo 2 


\section{Lista de Tabelas}

Tabela 1-50 Maiores Empresas da Indústria Farmacêutica Brasileira 58

Tabela 2-Testes de Normalidade das Variáveis 69

Tabela 3-Matriz de Correlação das Variáveis Estratégicas $\quad 71$

Tabela 4-Matriz de Correlação das Variáveis

de Desempenho 73

Tabela 5-Resultado da Análise de Fatores $\quad 74$

Tabela 6-Carga Final dos Fatores Após Rotação dos Eixos $\quad 75$

Tabela 7-Fatores Resultantes da Redução do Espaço

Estratégico

77

Tabela 8-Percentis Utilizados na Montagem dos

Centróides Iniciais

78

Tabela 9-Matriz dos Centróides Iniciais dos Sete Grupos

Estratégicos-Distribuição dos Percentis Teóricos

Tabela 10-Matriz dos Centróides Iniciais dos Sete Grupos

Estratégicos-Distribuição dos Percentis Aplicados

Tabela 11-Matriz dos Centróides Finais dos Sete Grupos

Estratégicos

80

Tabela 12-Teste de Igualdade das Matrizes dos Centróides $\quad 80$

Tabela 13-Distâncias entre Centróides dos Clusters Finais $\quad 82$

Tabela 14-Teste de Igualdade dos Centróides 83

Tabela 15-Grupos Estratégicos Formados à Luz da

Teoria de Miller e Dess 84

Tabela 16-Comparação de Variáveis por Grupo Estratégico $\quad 85$

Tabela 17-Empresas do Grupo I 85

Tabela 18-Empresas do Grupo II 87

Tabela 19-Empresas do Grupo III 88

Tabela 20-Empresas do Grupo IV 88

Tabela 21-Empresas do Grupo V 89

Tabela 22-Empresas do Grupo VI 90 
Tabela 23-Empresas do Grupo VII

Tabela 24-Equivalência dos Grupos Estratégicos

Formados nas Tipologias de Miller e Dess e Porter

92

Tabela 25-Grupos das Empresas nas Tipologias de

Miller e Dess e Porter

Tabela 26-Estatísticas de Desempenho por Grupo

Estratégico

Tabela 27-Teste de Igualdade de Desempenho dos

Grupos Estratégicos

Tabela 28-Ordenação das Médias de Desempenho por

Grupo Estratégico

Tabela 29-Comparação de Desempenho Entre Grupos

Estratégicos

Tabela 30-Posicionamento das Empresas do Grupo I 102

Tabela 31-Posicionamento das Empresas do Grupo II 103

Tabela 32-Posicionamento das Empresas do Grupo III 104

Tabela 33-Posicionamento das Empresas do Grupo IV 105

Tabela 34-Posicionamento das Empresas do Grupo V 106

Tabela 35-Posicionamento das Empresas do Grupo VI 107

Tabela 36-Posicionamento das Empresas do Grupo VII 108

Tabela 37-Empresas Agrupadas pelo Posicionamento

Competitivo

109

Tabela 38-Estatísticas de Desempenho Dentro dos

Grupos Estratégicos

Tabela 39-Testes de Igualdade de Desempenho das Empresas Primárias e Secundárias Dentro dos Grupos Estratégicos

Tabela 40-Estatísticas de Desempenho Dentro da Indústria Farmacêutica

Tabela 41-Testes de Igualdade de Desempenho das

Empresas Primárias, Secundárias e Solitárias Dentro

da Indústria Farmacêutica

Tabela 42-Análise de Regressão do Modelo 1

Tabela 43-Análise de Regressão do Modelo 2 


\section{Lista de Figuras}

Figura 1-Modelo das Cinco Forças de Porter 21

Figura 2-Estratégias Genéricas de Porter 35

Figura 3-Os Sete Tipos Estratégicos de Miller e Dess 41

Figura 4-Scatterplot dos Resíduos do Modelo $1 \quad 116$

Figura 5-Scatterplot dos Resíduos do Modelo $2 \quad 118$ 